\title{
Concurrent loss of heterozygosity and copy number analysis in adenoid cystic carcinoma by SNP genotyping arrays
}

\author{
Yongtao $\mathrm{Yu}^{1}$, Alexander S Baras ${ }^{1}$, Kanemitsu Shirasuna ${ }^{2}$, Henry F Frierson $\mathrm{Jr}^{1}$ and Christopher A Moskaluk ${ }^{1,3}$
}

Adenoid cystic carcinoma (ACC) is one of the most common malignancies to arise in the salivary glands, yet very little is known of the genetic alterations that are involved in the pathogenesis of this disease. To further examine the genetic changes that underlie ACC, we analyzed genomic DNA obtained from 22 primary ACC and two ACC-derived cell lines by high-density oligonucleotide single-nucleotide polymorphism genotyping arrays (Affymetrix GeneChip ${ }^{\circledR}$ Human Mapping 100K Set). Allelotype calls were analyzed by the Haplotype Correction version of the Linkage Disequilibrium Hidden Markov Model to determine loss of heterozygosity using information derived only from tumor samples. Comparison of data obtained from matched tumor-normal samples suggested that only deletion calls of $>3 \mathrm{Mb}$ were reliable. Within these parameters, ACC samples revealed a mean of three deletions per tumor, and no consensus areas of deletion were observed across the majority of tumors. Similarly, copy number analysis of primary hybridization data revealed no consensus areas of gene amplification. This is in contrast to a much higher rate of genomic alterations detected in a cohort of squamous carcinomas analyzed by the same methods. Our data show that most ACC have predominantly stable genomes, which is consistent with the theory that telomere crisis does not play a significant role in early stages of ACC tumor progression. Our data suggest that gene mutation and/or epigenetic events that cannot be detected by assay of gross alteration of chromosomal structure are likely to underlie the malignant transformation events of this tumor type. Laboratory Investigation (2007) 87, 430-439. doi:10.1038/labinvest.3700536; published online 19 March 2007

KEYWORDS: adenoid cystic carcinoma; single-nucleotide polymorphism; loss of heterozygosity; gene copy number; microarray

Salivary gland tumors are highly heterogeneous neoplasms, with a variety of histological appearances and clinical behaviors. The predominant benign salivary gland tumor is pleomorphic adenoma whereas the most frequent malignant lesions include mucoepidermoid carcinoma and adenoid cystic carcinoma (ACC). ${ }^{1}$ ACC exhibits myoepithelial differentiation, secretes copious amounts of basement membrane components, has a proclivity for nerve invasion, rarely metastasizes to lymph nodes, and has a slow but relentless clinical course with most disease-specific mortality occurring more than 5 years after diagnosis. ${ }^{1,2}$ ACC usually arises in major or minor salivary glands, but can also develop in related glandular sites (peribronchial and lacrimal glands) as well as in some extra-salivary locations such as the breast and vulva.

Previous investigations attempting to identify genetic changes occurring in ACC have included cytogenetic stu- dies, ${ }^{3-8}$ fluorescence in situ hybridization, ${ }^{9,10}$ comparative genomic hybridization $(\mathrm{CGH}),{ }^{11}$ and microsatellite loss of heterozygosity (LOH) analyses. ${ }^{12-15}$ Although these studies have indicated that portions of chromosomes 6, 12, 13, and 19 have the highest rates of alteration in ACC, a specific oncogene or tumor suppressor gene has not been definitively shown to be involved in the pathogenesis of this tumor type.

Recently, oligonucleotide microarrays that query singlenucleotide polymorphisms (SNPs) have been developed to globally analyze the genomes of cancers for genetic alterations. SNPs are the most common form of sequence variation in the human genome, occurring approximately every $1200 \mathrm{bp}$. The loss of heterozygous alleles at SNP loci in tumor specimens can be used in a manner analogous to the traditional forms of LOH mapping of restriction fragment polymorphisms or microsatellite repeats. However, the

\footnotetext{
${ }^{1}$ Department of Pathology, University of Virginia, Charlottesville, VA, USA; ${ }^{2}$ Department of Oral and Maxillofacial Surgery, Graduate School of Dental Science, Kyushu University, Fukuoka, Japan and ${ }^{3}$ Department of Biochemistry and Molecular Genetics, University of Virginia, Charlottesville, VA, USA

Correspondence: Dr C Moskaluk, MD, PhD, Department of Pathology, Health Sciences Center, University of Virginia, PO Box 800214, Charlottesville, VA 22908, USA. E-mail: cam5p@virginia.edu
} 
high density of SNPs and the high throughput format of microarrays allow a great density of mapping data to be generated in a short time interval. In addition, the hybridization intensity of probes generated from tumor genomes can be used to derive relative copy number in addition to specific sequence information. ${ }^{16,17}$ Another recent advance in the use of this technology is the development of the Haplotype Correction version of the Linkage Disequilibrium Hidden Markov Model (HC/LD-HMM) that allows the use of high-density oligonucleotide SNP array data for the accurate identification of chromosomal deletion in tumors without the need for paired normal samples. ${ }^{18}$

Gross alterations in chromosomal structure have been used in the analysis of other tumor types to identify areas of genomic loss consistent with tumor suppressor inactivation or to identify areas of high-level genomic amplification consistent with oncogene activation. Given the relative paucity of knowledge about the specific genetic alterations in ACC, and that SNP microarray analysis has not been reported for this tumor type, we have used this method to query the genetic changes in a cohort of 22 ACC.

\section{MATERIALS AND METHODS}

\section{Primary Tumor and Cell Line Specimens}

Approval for the present study was obtained from the Institutional Review Board of the University of Virginia. Samples were obtained from discarded tissue of surgical resection specimens at the University of Virginia Health System and of the Cooperative Human Tissue Network (http:// www.chtn.ims.nci.nih.gov/index.html). Twenty-two tumor and nine matching non-neoplastic tissue samples were obtained; the clinicopathologic features of these cases are shown in Table 1 . These samples were promptly frozen at $-80^{\circ} \mathrm{C}$ or in liquid nitrogen after initial gross examination. Frozen tissue samples were subsequently embedded in OCT and frozen histologic sectioning was performed. Tumors were graded as lesions with no solid component (grade I), $<30 \%$ solid areas (grade II) and $>30 \%$ solid component (grade III). ${ }^{19}$

As controls for the ACC tumors, which predominantly arose in the head and neck region, frozen samples of squamous carcinomas arising in the same region were similarly obtained and processed.

The ACC3 and ACCS cell lines have been previously established from primary salivary ACC. ${ }^{20,21}$ ACC3 cells were kindly provided by Dr Takashi Saku of Niigata University School of Dentistry, Japan and were cultured in RPMI 1640 with $10 \%$ fetal calf serum, $2 \mathrm{mM}$ glutamine at $37^{\circ} \mathrm{C}$ in a humidified $5 \% \mathrm{CO}_{2}$ incubator. ACCS cells were kindly provided by Dr Kanemitsu Shirasuna of Kyushu University, Japan and were cultured in DMEM with $10 \%$ fetal calf serum containing glucose and L-glutamine at $37^{\circ} \mathrm{C}$ in a humidified $5 \% \mathrm{CO}_{2}$ incubator.
Table 1 Clinicopathologic characteristics of ACC

\begin{tabular}{|c|c|c|c|c|c|}
\hline $\begin{array}{l}\text { ACC } \\
\text { case no. }\end{array}$ & Age & Gender & $\begin{array}{l}\text { Site of } \\
\text { primary tumor }\end{array}$ & Metastatic & $\begin{array}{l}\text { Histologic } \\
\text { grade }\end{array}$ \\
\hline 1 & 43 & $\mathrm{~F}$ & Trachea & $\mathrm{N}$ & 1 \\
\hline 2 & 42 & M & Larynx & $\mathrm{N}$ & 3 \\
\hline 3 & 26 & M & Parotid & $\mathrm{N}$ & 1 \\
\hline 4 & 53 & M & Nasal cavity & $\mathrm{N}$ & 1 \\
\hline 6 & 62 & M & Parotid & $\mathrm{N}$ & 3 \\
\hline 7 & 33 & M & Parotid & $\mathrm{N}$ & 1 \\
\hline 8 & 51 & M & Nasal cavity & $\mathrm{N}$ & 1 \\
\hline 9 & 54 & $\mathrm{~F}$ & Palate & $\mathrm{N}$ & 1 \\
\hline 10 & 77 & $\mathrm{~F}$ & Parotid & $\mathrm{N}$ & 3 \\
\hline 11 & 70 & $\mathrm{~F}$ & Oral cavity & $\mathrm{N}$ & 1 \\
\hline 12 & 54 & $M$ & Oral cavity & $\mathrm{N}$ & 2 \\
\hline 13 & 61 & M & Parotid & $\mathrm{N}$ & 1 \\
\hline 14 & 65 & M & Oral cavity & $\mathrm{N}$ & 2 \\
\hline 15 & 30 & M & Unknown & Unknown & 1 \\
\hline 16 & 82 & $\mathrm{~F}$ & Nasal cavity & $\mathrm{N}$ & 1 \\
\hline 17 & 61 & M & Oral cavity & $\mathrm{N}$ & 1 \\
\hline 18 & 76 & $M$ & Oral cavity & Y (kidney) & 1 \\
\hline 20 & 42 & $\mathrm{~F}$ & Parotid & $\mathrm{N}$ & 1 \\
\hline 21 & 61 & $\mathrm{~F}$ & Parotid & $\mathrm{N}$ & 1 \\
\hline 24 & 23 & $\mathrm{~F}$ & Parotid & $\mathrm{N}$ & 1 \\
\hline 29 & 44 & M & Parotid & Y (lung) & 1 \\
\hline 31 & 45 & $\mathrm{~F}$ & Unknown & Y (lung) & 1 \\
\hline
\end{tabular}

\section{Macrodissection of Tissue Samples and Isolation of Genomic DNA}

Areas containing $>80 \%$ tumor cells were circled on the frozen histologic sections, which were used as guides to gross dissect regions of the frozen tissue using a scalpel blade. Normal tissues free of tumor were similarly selected and dissected. Tissue was disrupted using a Pro-200 tissue homogenizer (ProScientific, Monroe, CT, USA). DNA was isolated from tumor/normal tissue or cell pellets by proteinase $\mathrm{K}$ digestion followed by phenol/chloroform/isoamyl alcohol extraction and ethanol precipitation. DNA concentration was determined by spectrophotometric absorbance at $260 \mathrm{~nm}$ wavelength.

\section{DNA Probe Preparation and SNP Microarray Hybridization}

DNA from normal and cancer samples was prepared for microarray hybridization using the GeneChip ${ }^{\circledR}$ Mapping Assay Protocol (Affymetrix Inc., Santa Clara, CA, USA). Briefly, $250 \mathrm{ng}$ total genomic DNA was digested with XbaI or HindIII and ligated to adaptors that hybridize to the $4 \mathrm{bp}$ overhangs. A generic primer that recognizes the adaptor 
sequence was used to preferentially amplify adaptor-ligated DNA fragments in the 250-2000 bp size range by optimized PCR conditions. The amplified DNA was then fragmented by DNase, labeled, and hybridized to the Affymetrix GeneChip ${ }^{\mathbb{R}}$ Human Mapping 100K Set. Hybridization, washing, staining, and chip scanning were performed by the University of Virginia Biomolecular Research Core Facility using materials and methods provided by the manufacturer (Affymetrix Inc.).

\section{Imaging and Data Analysis}

The following analysis methods were implemented in the software dChip 2006 (DNA-Chip Analyzer) (Build date: 7 July 2006), ${ }^{22}$ which is available online to academic users (http://biosun1.harvard.edu/ cli/dchip2006.exe).

\section{Normalization of arrays and model-based signal values}

Normalization of array fluorescence intensity data and generation of probe-specific signal value were performed using the perfect match/mismatch (PM/MM) difference model ${ }^{23}$ in dChip 2006 (http://biosun1.harvard.edu/complab/dchip/ manual.htm).

\section{Loss of heterozygosity analysis in paired normal/tumor} samples and in tumor-only samples

Features used in the dChip 2006 software included automated reading of SNP calls from SNP call text files, pooling SNP array replicates, making $\mathrm{LOH}$ calls based on comparison of paired tumor/normal samples, making statistical inference for identifying shared LOH regions, copy number analysis (CNA) of SNP array, and sample clustering by $\mathrm{LOH}$ profiles. $\mathrm{LOH}$ regions in paired tumor/normal samples were detected and demarcated using a Hidden Markov Model (HMM), as described previously. ${ }^{18,22}$ One advantage of this approach is to help suppress the effects of genotyping errors in high-density microarray data. For $\mathrm{LOH}$ analysis using SNP call data only from tumor samples, the HC/LD-HMM was employed. ${ }^{18}$ This method predicts the presence of deletions based on the prevalence of homozygosity at SNP alleles in the tumor population as compared with the prevalence of homozygosity in a reference population. As the majority of samples in our tumor cohort were obtained from Caucasians, data from a reference population of 60 Caucasians available from the CEPH cohort were used.

Both methods return $\mathrm{LOH}$ prevalence scores, measuring the prevalence of $\mathrm{LOH}$ at each marker in the entire cohort, computed as the average probability of $\mathrm{LOH}$. As the threshold cutoff for a consensus LOH call is dependent on the number of samples in the analysis, these were determined empirically after examination of the data for the various analyses. For the analysis of the nine paired ACC tumor/ normal samples, LOH prevalence scores above 0.095 were considered consensus $\mathrm{LOH}$ areas, and for the analysis of the 22 unpaired ACC tumor samples, the threshold was set at 0.042. The inferred SNP LOH calls for each case were exported to Excel spreadsheets (Microsoft Inc., Redmond, WA, USA) along with annotated SNP mapping data. In this manner, the size and boundaries of areas of contiguous SNP LOH calls were determined for individual tumors.

\section{Copy number analysis}

DNA copy number was determined by the hybridization intensity of each SNP probe. The signal intensities of the tumor sample were compared with the nine matching nonneoplastic tissue samples and with a data set obtained from non-neoplastic DNA samples of 12 other individuals (obtained from Affymetrix). From raw signal data, the inferred copy numbers at each SNP locus were estimated by HMM analysis. ${ }^{16}$ The dChip software was then used to visualize and map the LOH and copy number abnormality (CNA) patterns for each tumor sample. To determine the fluorescence intensity that indicates normal copy number for any individual SNP, $5 \%$ of the values with extreme signals from each end of the spectrum were trimmed to remove potential technical artifacts, and the remaining values were used to estimate the mean and s.d. of the signal distribution.

\section{Microsatellite Loss of Heterozygosity Assays}

MapPair $^{\mathrm{TM}}$ primers for eight microsatellite markers (D1S2888, D1S429, D1S2881, D1S2852, D1S2687, D1S189, D1S453 and D1S2669) were obtained from Invitrogen (Carlsbad, CA, USA). Radiolabeled PCR amplification, gel electrophoresis, autoradiography of microsatellite markers and $\mathrm{LOH}$ determination were performed as described previously. ${ }^{24}$

\section{DNA Sequencing}

PCR amplification of individual exons of the CDKN2A/p16 gene was performed using conditions described previously. ${ }^{25}$ Sequencing was performed on an Applied Biosystems 377 Prism ${ }^{\circledR}$ DNA Sequencer, using BigDye ${ }^{\circledR}$ terminator chemistry with Taq DNA polymerase. PCR and sequencing primers are available upon request. Automated DNA sequence calls were analyzed by BLAST, followed by manual review of chromatograms.

\section{Statistical Analysis}

Comparison of deletion events between ACC of varying histologic grade and between the ACC and squamous cell carcinoma (SCC) cohorts was performed using unpaired $t$-tests (two-tailed) with Welch's correction. All calculations were performed in the Prism software suite, Version 4.0a for Macintosh (GraphPad Software Inc.).

\section{RESULTS}

\section{SNP Array Hybridization}

Twenty-two ACC tumor samples, nine matching non-neoplastic tissue samples, and two ACC cell lines (ACC3 and 
ACCS) were assayed for $\mathrm{LOH}$ and copy number alterations using the Affymetrix GeneChip ${ }^{\circledR}$ Human Mapping 100K Set SNP chips representing 116204 SNPs. The overall SNP call rate for the cohort was $94.30 \%$ and ranged from 80.70 to $99.05 \%$. The average call rate did not vary significantly between the ACC tumors $(94.6 \pm 4.0 \%)$ and matching normal tissue $(92.9 \pm 2.5 \%)$.

\section{Genome-Wide LOH Analysis of ACC}

HMM analysis was used to infer the probability of $\mathrm{LOH}$ based on the observed $\mathrm{LOH}$ calls from the nine paired nor$\mathrm{mal} /$ tumor samples. Thirty-five areas of deletion were identified, ranging in size from 0.05 to $135.87 \mathrm{Mb}$. Twelve of these $\mathrm{LOH}$ areas represented large portions of the chromosomes $(>3 \mathrm{Mb})$. Twelve of the 23 smaller $\mathrm{LOH}$ areas $(<3 \mathrm{Mb})$ came from clustered areas of deletion on just two chromosomes from two cases, chromosome 5 in case 10 and chromosome 14 in case 31 (Figure 1).

As other tumor samples in our cohort and the ACC cell lines lacked matching normal DNA samples, HC/LD-HMM analysis was used to generate inferred $\mathrm{LOH}$ areas from the nine tumor samples only, which had previously been analyzed together with their matched non-neoplastic samples. Fifty-two deletion areas were identified by the HC/LD-HMM analysis. Figure 1 shows the graphical comparison of deletion events found in these nine samples by analyzing the paired samples by HMM and the tumor samples alone by HC/LDHMM. By visual inspection, there is excellent correlation between the two methods in identifying large deletions. However, it is clear that the HC/LD-HMM analysis generated many more $\mathrm{LOH}$ calls of small size. By comparing the $\mathrm{LOH}$ areas by the two methods, we found that there was perfect correlation for deletions $\geq 3 \mathrm{Mb}$ (12 of 12). Of the remaining deletions less than $3 \mathrm{Mb}$ in size ( 23 detected by HMM analysis of matched samples, 40 detected by HC/LD-HMM analysis of tumor samples alone), there were only two matches. As HMM analysis of paired tumor/normal samples suppresses false positive calls on the basis of contiguous stretches of homozygosity unrelated to chromosomal deletion events, it was concluded that the majority of $\mathrm{LOH}$ calls by $\mathrm{HC} / \mathrm{LD}-\mathrm{HMM}$ that are $<3 \mathrm{Mb}$ in size are false positive results.
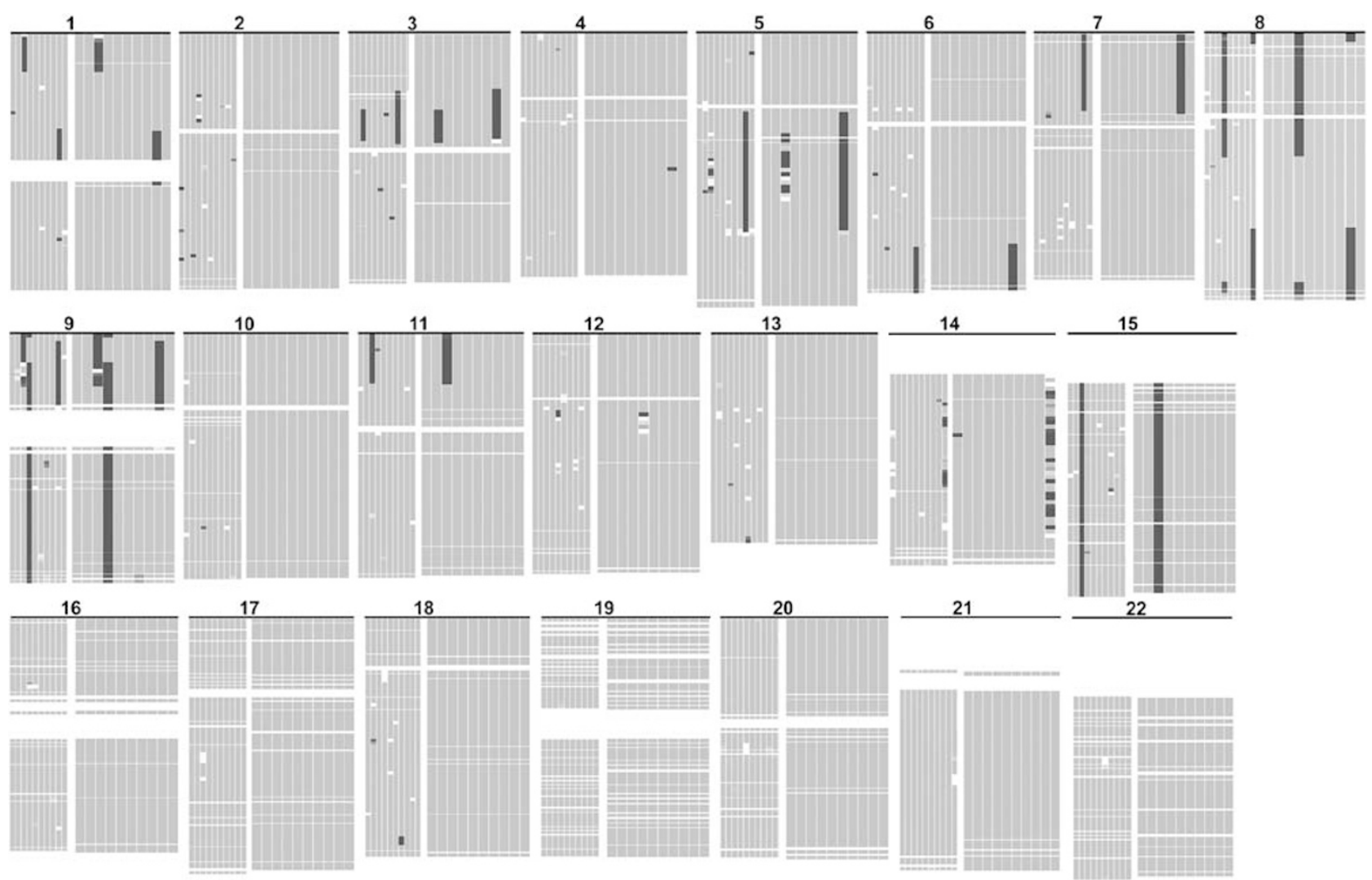

Figure 1 Comparison of LOH results between HMM analysis of matched tumor/normal DNA samples vs HC/LD-HMM analysis of tumor DNA samples. Each autosomal chromosome for each case is represented by a gray column. Under each chromosome number are two panels; the left-hand panel represents HC/LD-HMM analysis of tumor samples alone, and the right-hand panel represents HMM analysis comparing tumor and normal samples. In each panel the cases are arranged going from left to right in the following order: 1, 8, 10,11, 12, 13, 18, 24, 29, and 31. Black areas within the columns represent areas of LOH calls. White areas common to the cases are chromosomal areas without SNP representation (usually centromere regions). White areas specific to individual cases represent zones of 'no calls'. Comparing the two methods shows that large deletion areas are reproducibly detected by the HC/LD-HMM analysis, but that detection of small areas of deletion $(<3 \mathrm{Mb})$ are not reproducible between the two methods. 
Ignoring $\mathrm{LOH}$ calls of $<3 \mathrm{Mb}$, the entire ACC tumor cohort was analyzed by HC/LD-HMM. Fifty large LOH areas were found within these ACC tumor samples, with sizes ranging from 3.0 to $122.1 \mathrm{Mb}$ (Figure 2). The average number of deletions per tumor sample was 2.3. The cell lines showed a greater number and size of genetic deletions than the primary tumors, with an average of 14.5 deletions found per cell line.

To validate the presence of chromosomal deletions detected by SNP GeneChip, we performed microsatellite PCR $\mathrm{LOH}$ analysis in a subset of samples, using microsatellite alleles that mapped to the areas predicted to be deleted. Only one large deletion area from one tumor sample was adequate

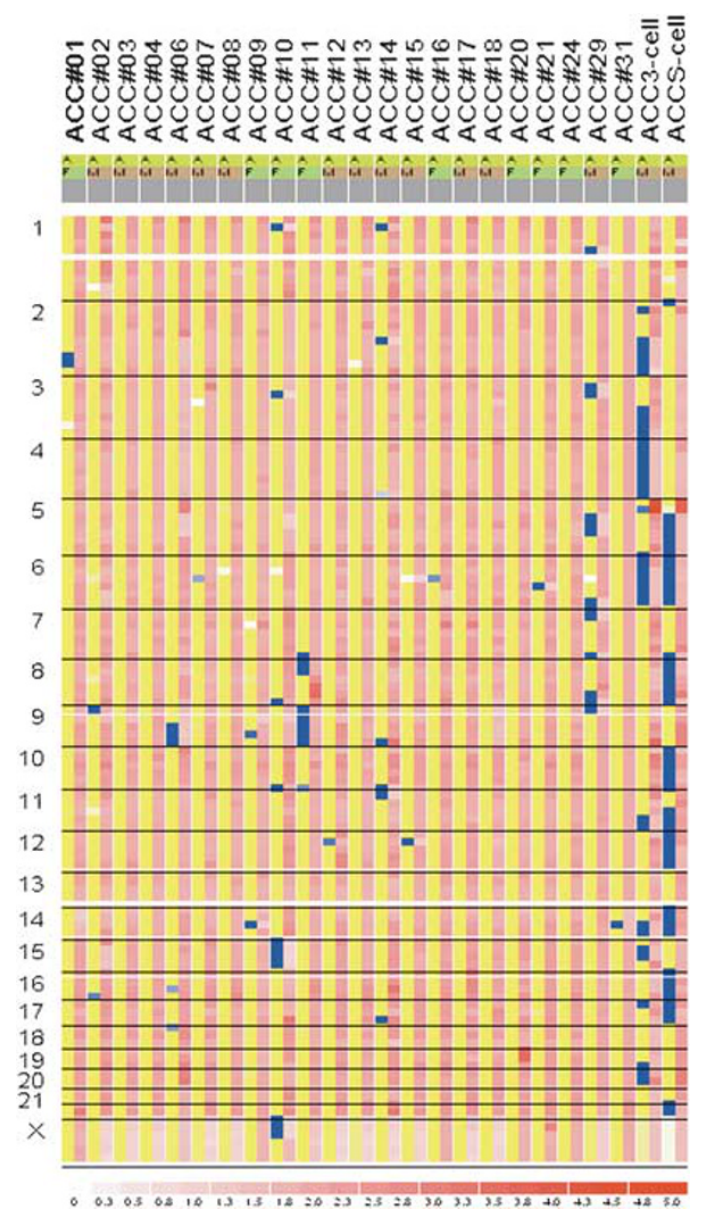

Figure 2 HC/LD-HMM LOH and CNA in ACC. The figure shows graphical representations of the analysis of SNP array data for 22 ACC tumor samples and two ACC-derived cell lines (ACC3 and ACCS). Each sample is represented by two adjacent columns (LOH column on the left, CNA column on the right). The vertical axis of each column represents the genetic location of the SNP markers, with chromosomal localization indicated on the left-hand side of the figure. Color code of $\mathrm{LOH}$ column: inferred probability of LOH is displayed from blue (1) to white $(0.5)$ to yellow (0). Copy number is represented by different intensity of red as indicated in the color bar at the bottom of the figure. Lighter shades of red indicate loss of copy number and darker shades of red indicate gain of copy number Additional information on the areas of $\mathrm{LOH}$ can be found in Supplementary Table 1. for this analysis, as more than half of the tumor samples with LOH detected by the SNP GeneChip analysis did not have matching normal control tissue, some of the remaining candidate LOH areas detected by the SNP GeneChip had no microsatellite makers mapped within them or had markers with low rates of heterozygosity; most of the remaining candidate LOH areas detected by the SNP GeneChip analysis only had single suitable microsatellite makers located at one extreme end of the deletion area. In the deletion area that was analyzed by microsatellite PCR, seven informative microsatellite markers showed LOH, corroborating the SNP GeneChip LOH analysis (Figure 3).

The most frequent area of deletion was on chromosome $9 \mathrm{p}$ (five of 22 tumors), which includes the locus of the CDKN2A/p16 tumor suppressor gene (four of 22 tumors) (Figure 4). We analyzed the DNA sequence of this gene in the four tumor samples that had $\mathrm{LOH}$ spanning the p16

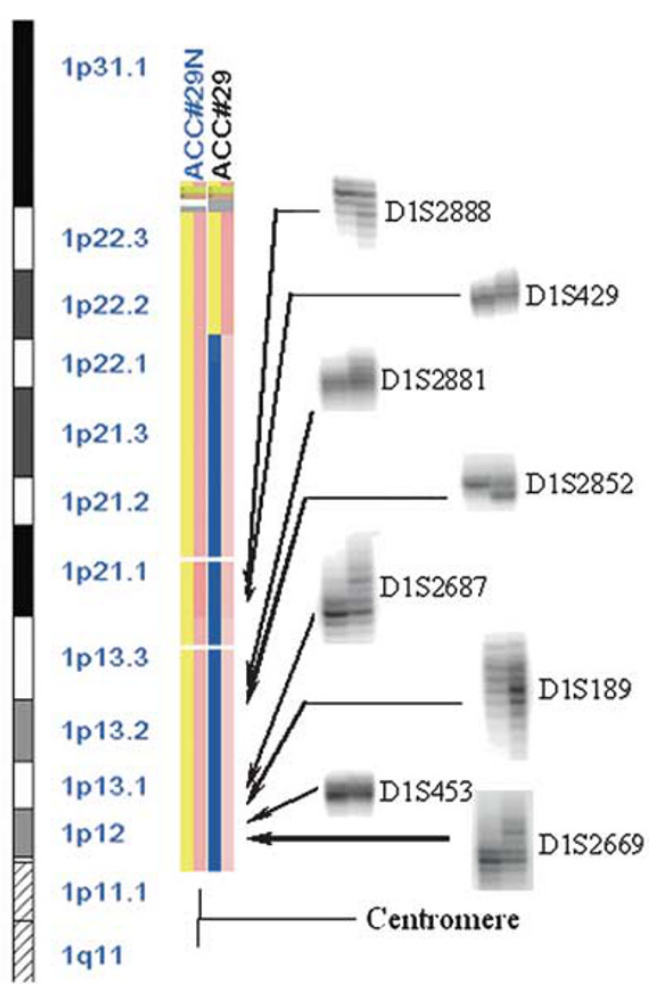

Figure 3 Validation of SNP Array LOH Call by Microsatellite PCR Analysis. An area of deletion detected in an ACC tumor sample (ACC no. 29) by SNP GeneChip analysis is shown. The GeneChip results for both the tumor sample and the sample of matched non-neoplastic tissue (ACC no. 29N) are represented by two adjacent columns ( $\mathrm{LOH}$ column on the left, CNA column on the right), with the same color codes as in Figure 2. The GeneChip data shows a genotype call of LOH in the tumor sample (blue color in the left-hand column) that corresponds to a loss of copy number (area of lighter red color in the right-hand column). The results of the microsatellite PCR assays are shown in labeled boxes in the right-hand portion of the figure, with PCR products from tumor DNA in the left lanes, and PCR product from matching normal DNA in the right lanes. The marker D1S453 is non-informative. All other microsatellite markers are informative and show the loss of an allele in the tumor samples. 


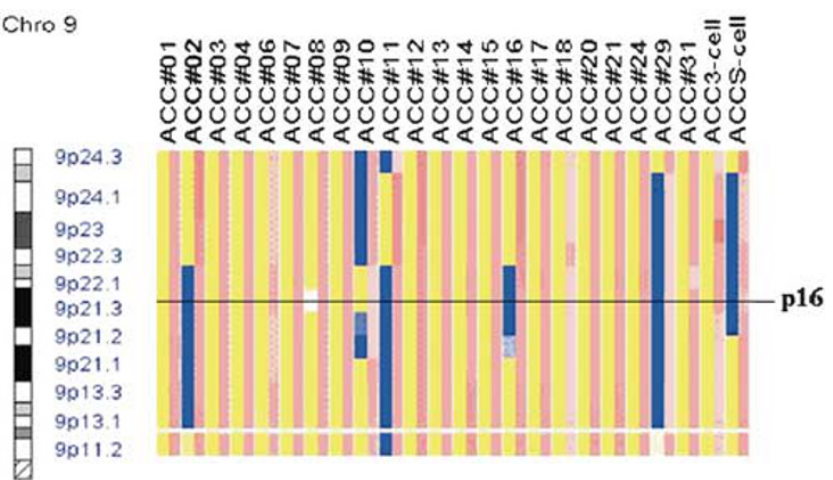

Figure 4 Chromosome 9p LOH in ACC. Five ACC tumors had areas of deletion on chromosome $9 p$ detected in this analysis, four of the five included the locus of the CDKN2A/p16 tumor suppressor gene (shown by the black line). The LOH and CNA data are presented in columns, with the same color codes as in Figure 2.

locus and in the two ACC-derived cell lines, and found no mutations.

We compared the number of $\mathrm{LOH}$ calls to the histologic grade of the tumors. Most of the tumors in our cohort were of low histologic grade (17 of 22 were Grade 1 tumors). Although there was a trend for tumors of higher histologic grade to have more deletions greater than $3 \mathrm{Mb}$ in size (mean of six deletions for Grade 2 and three tumors $v s$ mean of two deletions for Grade 1 tumors). However, this trend did not reach statistical significance $(P=0.1095)$, and analysis of more higher histologic grade tumors will be probably required to determine if higher histologic grade ACC have a greater degree of genomic deletion than low-histologic grade tumors.

To determine if the relatively low rate of genetic deletion was due to insensitivity of the analytic method or was a true reflection of the relative paucity of genetic aberrations in ACC, we performed the same analysis in 22 SCC samples arising in the head and neck region. SCC are known to have unstable genomes as detected by a variety of methods. ${ }^{26,27}$ Compared to the mean of 2.3 deletions per tumor sample in the ACC cohort, the SCC samples showed a mean number of 20.7 deletions, including several loci that were deleted in more than half of the cases (Figure 5). Although the details of this SCC analysis will be published elsewhere, frequent deletions in chromosomes 3p25, 3p21.3, 9q11-q21, 9q33-q34, 13q13.1, 13q14.2 and 17q25 were identified, which correlate to findings of previous studies of SCC, ${ }^{28-33}$ further validating our application of SNP genotyping arrays to these tumor cohorts. These results suggest that the paucity of genetic deletions identified in ACC by our SNP array analysis is a reflection of the relative genomic stability of this tumor type.

\section{Copy Number Analysis in ACC}

The fluorescence intensity values obtained from the SNP array hybridization were used to predict CNA. Concurrent genome-wide LOH and CNA results are shown in Figure 1.
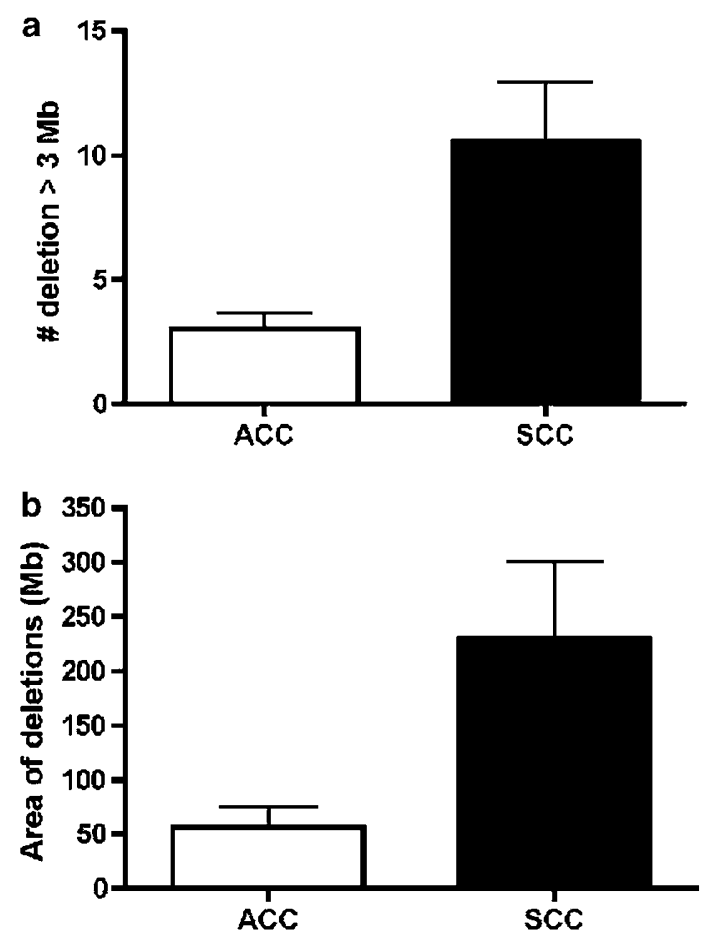

Figure 5 Comparison of SNP array LOH analysis for ACC and SCC. Twentytwo samples of SCC arising in the head and neck region were processed and analyzed identically to the ACC cohort. (a) The mean number of $\mathrm{LOH}$ events per sample is shown with 95\% confidence intervals (error bars) for the two tumor types. (b) The average genomic area (in Mb) involved in the LOH events per sample is shown with $95 \%$ confidence intervals (error bars) for the two tumor types. There is significantly less LOH in ACC than in SCC, in both numbers of and size of deletion events.

Twenty-eight of 82 (34\%) of LOH events had copy number reduction, consistent with these $\mathrm{LOH}$ events being caused by hemizygous chromosomal deletion. The remaining examples of LOH without copy number change or with copy number increase could be caused by copy-neutral events such as mitotic nondisjunction followed by duplication of one of the parental chromosomes. ${ }^{16}$

Areas of copy number $<1.5$, inferring gene copy number loss, were found in less than $20 \%$ of samples (Figure 6). Similar to the LOH profile determined by HC/LD-HMM, no major consensus copy number loss was found in ACC. Thus, two parameters determined from the SNP GeneChip hybridization analysis, genotype and DNA copy number, demonstrate relative stability of the genomes in ACC.

A copy number level of three in at least two contiguous SNPs was used as a cutoff to identify potential areas of genomic amplification. Using this cutoff, there were no amplified areas that were shared by more than two tumors. Using a more stringent criterion of a copy number of 6 in at least two contiguous SNPs, we identified only two potential areas of high copy number gain in the ACC 3 cell line. One high level amplification spanning $0.78 \mathrm{Mb}$ on chromosome 7 p11.2 was represented by 63 contiguous SNPs, another one 


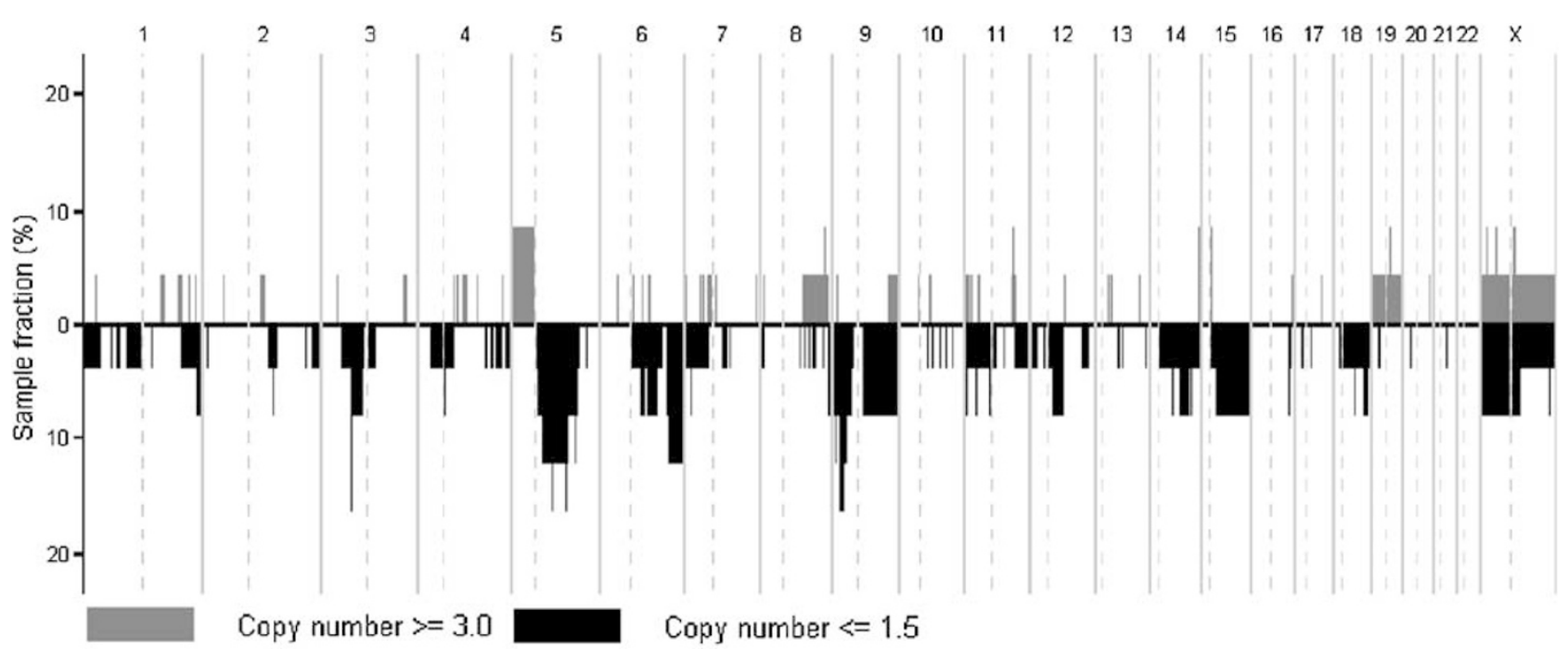

Figure 6 DNA copy number alterations in ACC by SNP array analysis. The fraction of samples with copy number amplification of at least three copies (gray bars), and copy number reduction to $<1.5$ copies (black bars) across all autosomal SNPs is shown for all of the ACC samples. SNP markers are ordered according to their mapped positions from chromosome 1 to $\mathrm{X}$ with vertical solid and dashed lines, indicating chromosome boundaries and centromeres, respectively. No area of chromosomal gain occurred in more than $10 \%$ of the tumor samples. No area of chromosomal loss was detected in more than $20 \%$ of the tumor samples.

spanning $0.4 \mathrm{~kb}$ on chromosome $2 \mathrm{p} 21$ was represented by two contiguous SNPs.

\section{DISCUSSION}

Deletion of chromosomal loci bearing tumor suppressor genes is a key event in the evolution of many epithelial and mesenchymal tumors. Identification of these regions usually relies on genotyping tumor and counterpart normal DNA, and noting regions where heterozygous alleles in the normal become homozygous in the tumor. Paired normal samples for tumors and cell lines are often not available, however, and mapping individual genetic loci by traditional methods is time consuming and laborious. The analysis of genomic DNA by high-density SNP microarrays with subsequent statistical analysis of contiguous genetic homozygosity offers a potential high throughput method for mapping genetic deletions in tumors alone without having matched non-neoplastic tissue samples.

As the Hidden Markov Model (HMM) determines a potential area of genetic deletion on the basis of homozygous calls in contiguous SNPs, false-positive calls can occur in genetic regions that contain SNPs with low rates of heterozygosity. This becomes more problematic as high-density platforms are employed, such as the Affymetrix 100K GeneChip array. HC/LD-HMM reduces the false LOH call from basic HMM, by removing false-positive $\mathrm{LOH}$ regions identified in a set of 60 normal reference samples. Despite this correction, there appears to be a high rate of false-positive calls for areas less than $3 \mathrm{Mb}$ in size when comparing results with the analysis from matched normal/tumor pairs. Thus, this methodology is useful in detecting relatively large areas of loss, but may miss smaller areas of deletion. Although we matched the predominantly Caucasian population in our sample cohort to 60 Caucasian subjects as the normal cohort, it is possible that variance in SNP heterozygosity between the test and reference populations plays a role in creating false positive results. In the original description of the HC/LD-HMM as implemented in dChip, major impact on specificity of LOH calls was not detected when reference and test populations were matched for race, although this analysis was performed with data using arrays with lower SNP density. ${ }^{18}$

Our current analysis supports the conclusion that no sizeable 'consensus' genetic deletion or amplification events occur in the majority of ACC. This result is consistent with accumulated data suggesting that this tumor tends to have karyotypes with few chromosomal alterations, ${ }^{3,4,7}$ a fact that has been supported by flow cytometry ploidy analysis. ${ }^{34-36}$ ACC is thus unlike most carcinomas, which tend to have marked aneusomy and complex chromosomal rearrangements thought to be secondary to telomere crisis, ${ }^{37}$ which results as a consequence of telomere shortening owing to reiterated rounds of cell replication early in the course of tumor progression. The fact that our methods detected a much higher rate of chromosomal deletion and CNAs in a cohort of squamous carcinomas of the head and neck region suggests that the observation of a paucity of genomic changes in ACC is not entirely a reflection of the lack of sensitivity of our methods. As ACC tumors typically have less intervening stroma than SCC, and given that our tumor tissues were chosen on the basis of high cellularity of neoplastic cells by histologic-guided dissection, these results can also not be explained by the trivial explanation of low tumor cellularity in our samples of ACC. 
Previous studies predominantly using PCR analysis of microsatellite markers to detect $\mathrm{LOH}$ in ACC have found chromosome 6 and 12 deletion at a higher rate than the current analysis (Table 2). Part of this discrepancy may be owing to the fact that the methodology employed in the SNP array analysis suppresses LOH calls from individual SNPs to dampen the effect of false-positive genotyping errors that complicate high-density array analysis. Conversely, single locus LOH events were reported as definitive calls in the previous work. This and the fact that $\mathrm{LOH}$ deletion calls of less than $3 \mathrm{Mb}$ are unreliable using the current SNP array analytic methods means that we could have missed small deletion events. Although it is possible that that there is a genomic locus in ACC that is preferentially targets by small deletions, most previous allelotyping studies in many tumor types suggest that for consensus areas of somatic deletion, a resolution of $3 \mathrm{Mb}$ is sufficient to identify greater than half of the events in a population to tumors. ${ }^{42-44}$ Another trivial explanation could be owing to the fact that the current tumor cohort collected had few high-grade tumors, and if there is a correlation between chromosomal alterations and tumor grade, our results could be biased by this selection. Although the overall percentage of tumors having $\mathrm{LOH}$ detected in these regions was not high, some of the most common $\mathrm{LOH}$ loci found in our analysis included the areas of $6 \mathrm{q}, 12 \mathrm{q}$, and $9 \mathrm{p}$, identified in previous karyotyping, microsatellite $\mathrm{LOH}$ and CGH analyses (Table 2). Previous cytogenetic analyses of ACC have typically involved small numbers of tumors, and a consensus reading of this literature identifies no specific abnormality that is present in the majority of tumors studied. However, the most frequent changes identified include chromosome 6q deletion, ${ }^{8,10,45}$ and translocations involving chromosomes 6q, 9p, and 12q. ${ }^{5,46}$ GTG-banding and cytophotometry analyses have found aneuploidy in some case of ACC ${ }^{34-36}$ but this too appears to occur in less than half of tumors so analyzed.

Although we identified $\mathrm{LOH}$ events that affected the $\mathrm{CDKN} 2 \mathrm{~A} / \mathrm{p} 16$ gene, we found no intragenic mutations in the remaining allele that could account for a genetic cause of biallelic inactivation. It has been well established that this gene

Table 2 Genomic changes previously reported in ACC

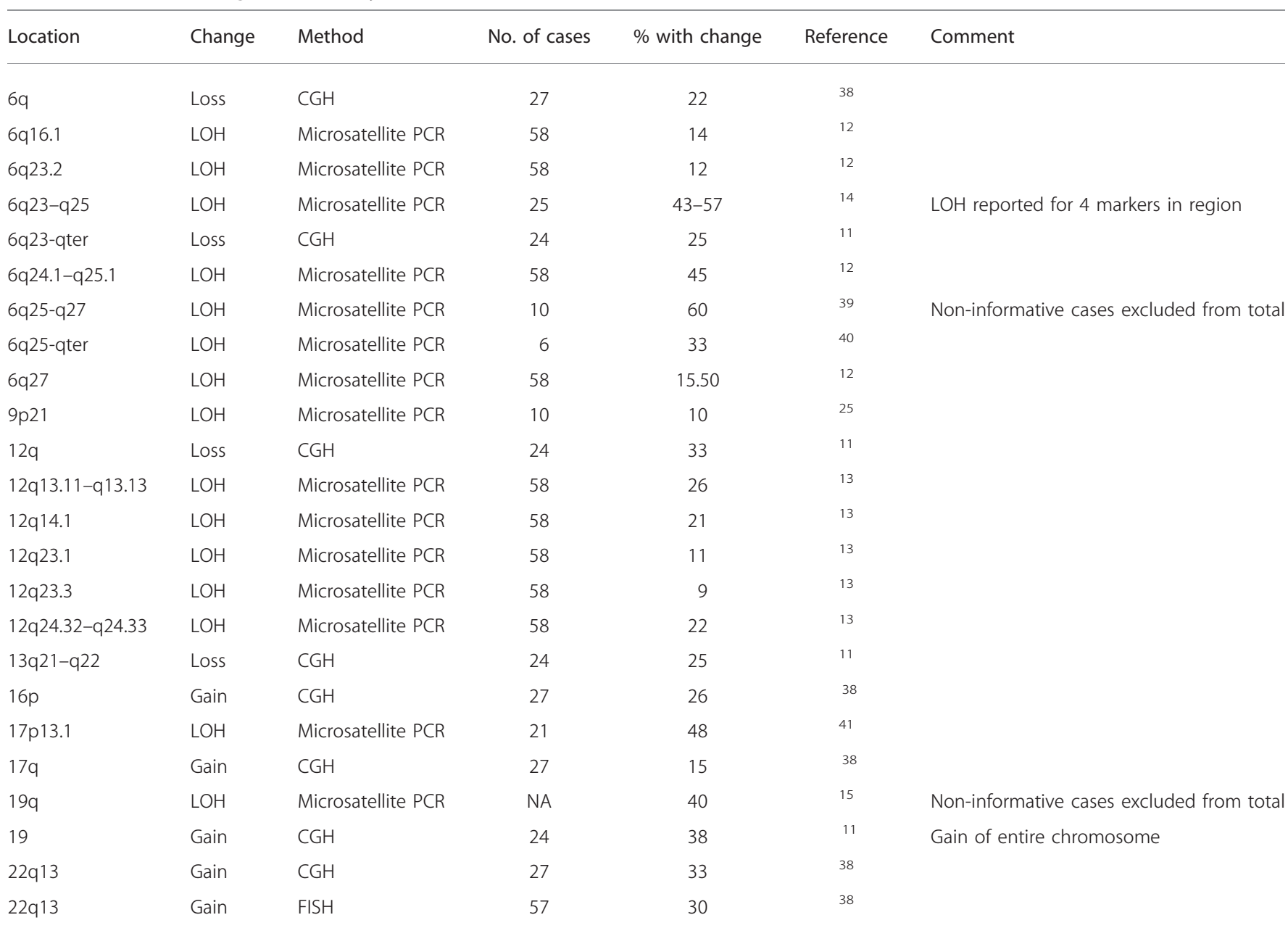


can be transcriptionally silenced, which may be an alternative mechanism of tumor suppression. In two previous studies, methylation of the p16 gene consistent with transcriptional silencing was found in 15 and $21 \%$ of ACC samples. ${ }^{47,48}$ Although we did not investigate gene expression levels in these tumor samples, this previous literature suggests that transcriptional silencing of p16 may play a role in a subset of ACC tumors.

It remains likely that one or a few specific genes are targeted to produce ACC. Although these genes may be affected at a low rate by gross chromosomal alterations, it would seem that small intragenic lesions or epigenetic regulatory events must be responsible for the majority of genetic dysregulation, at least in tumors of low histologic grade. These genetic or epigenetic events are not amenable to most methods employed in detecting gross genomic alterations.

We found a marked difference in the number and areas of genetic deletion between primary ACC and ACC cell lines. It is possible that ACC that have acquired a larger number of genetic alterations and have developed a phenotype of genomic instability and increased proliferation may be more amenable to growth in cell culture. Alternatively, it is possible that many of these changes occurred during the in vitro passaging of cell lines, with selection of clones that overgrew the original culture by the acquisition of new genetic changes. Obviously, the behavior of these cell culture models may not be reflective of primary ACC neoplasms.

Our data suggest that there may be a tendency for ACC of higher histologic grade to exhibit greater numbers and total size of genomic deletion. This is consistent with findings of greater genomic instability in high-grade ACC found by studies using comparative genomic hybridization ${ }^{11}$ and ploidy analysis. ${ }^{34,35}$ High histologic grade reflects a loss of differentiation and is associated with a more aggressive clinical course. ${ }^{1,19}$ However, most ACC present as low-grade neoplasms, though histologic progression has been documented in cases of recurrent tumors. ${ }^{49}$ These observations suggest that telomere crisis may occur in ACC tumor progression, but in a minority of cases and at late stages. This is in contrast to the current model for the role of telomere crisis as an early event in tumor progression for most carcinomas. ${ }^{37}$ It is therefore likely that high-grade ACC may acquire genetic lesions more typical of other carcinomas. Supporting evidence for this hypothesis is the increased frequency of p53 mutations in high-grade and/or recurrent $\mathrm{ACC}^{50-52}$ compared with low-grade neoplasms, and the evidence that p53 mutation is associated with telomere crisis in other types of carcinoma.

In conclusion, SNP array analysis of 22 primary ACC failed to detect high-level consensus areas of genetic loss or gain, and in general showed low levels of alterations in DNA copy number. These data support previous analyses using other methods, and suggest that most tumor-specific genetic changes important in the pathogenesis of ACC occur via mechanisms not detectable by assays of gross alterations in genomic structure.

Supplementary Information accompanies the paper on the Laboratory Investigation website (http://www.laboratoryinvestigation.org)

\section{ACKNOWLEDGEMENT}

We gratefully acknowledge the support of members of the Adenoid Cystic Carcinoma Registry at The University of Virginia (http:// www.healthsystem.virginia.edu/internet/cancer/teampages/moskaluk/ ACChome.cfm) for the donation of tumor samples and clinical data, and to the contributors to the Adenoid Cystic Carcinoma Research Fund at The University of Virginia. This work was supported by grants from the National Institute of Dental and Craniofacial Research (R01DE04694) and from Adenoid Cystic Carcinoma Organization International (http://www.orgsites.com/ca/acco/).

1. Zarbo RJ. Salivary gland neoplasia: a review for the practicing pathologist. Mod Pathol 2002;15:298-323.

2. Fordice J, Kershaw C, El-Naggar A, et al. Adenoid cystic carcinoma of the head and neck: predictors of morbidity and mortality. Arch Otolaryngol Head Neck Surg 1999;125:149-152.

3. Mark HF, Hanna I, Gnepp DR. Cytogenetic analysis of salivary gland type tumors. Oral Surg Oral Med Oral Pathol Oral Radiol Endod 1996;82:187-192.

4. Martins C, Fonseca I, Roque L, et al. Malignant salivary gland neoplasms: a cytogenetic study of 19 cases. Eur J Cancer B Oral Oncol 1996:32B:128-132.

5. Jin Y, Mertens F, Limon J, et al. Characteristic karyotypic features in lacrimal and salivary gland carcinomas. Br J Cancer 1994;70:42-47.

6. Nordkvist A, Mark J, Gustafsson H, et al. Non-random chromosome rearrangements in adenoid cystic carcinoma of the salivary glands. Genes Chromosomes Cancer 1994;10:115-121.

7. Sandros J, Stenman G, Mark J. Cytogenetic and molecular observations in human and experimental salivary gland tumors. Cancer Genet Cytogenet 1990;44:153-167.

8. Stenman G, Sandros J, Dahlenfors R, et al. $6 q$ - and loss of the $Y$ chromosome-two common deviations in malignant human salivary gland tumors. Cancer Genet Cytogenet 1986;22:283-293.

9. Roijer E, Dahlenfors R, Mark J, et al. Observations by chromosome banding, FISH and immunohistochemistry in an adenoid cystic carcinoma with del(17)(p13) as the sole deviation. Virchows Arch 1997:430:339-342.

10. Jin C, Martins C, Jin Y, et al. Characterization of chromosome aberrations in salivary gland tumors by $\mathrm{FISH}$, including multicolor COBRA-FISH. Genes Chromosomes Cancer 2001;30:161-167.

11. El-Rifai W, Rutherford S, Knuutila $S$, et al. Novel DNA copy number losses in chromosome 12q12-q13 in adenoid cystic carcinoma. Neoplasia 2001;3:173-178.

12. Rutherford $\mathrm{S}, \mathrm{Yu} Y$, Rumpel CA, et al. Chromosome 6 deletion and candidate tumor suppressor genes in adenoid cystic carcinoma. Cancer Lett 2006;236:309-317.

13. Rutherford S, Hampton GM, Frierson HF, et al. Mapping of candidate tumor suppressor genes on chromosome 12 in adenoid cystic carcinoma. Lab Invest 2005;85:1076-1085.

14. Stallmach $I$, Zenklusen $P$, Komminoth $P$, et al. Loss of heterozygosity at chromosome 6q23-25 correlates with clinical and histologic parameters in salivary gland adenoid cystic carcinoma. Virchows Arch 2002;440:77-84.

15. Johns III MM, Westra WH, Califano JA, et al. Allelotype of salivary gland tumors. Cancer Res 1996;56:1151-1154.

16. Zhao X, Li C, Paez JG, et al. An integrated view of copy number and allelic alterations in the cancer genome using single nucleotide polymorphism arrays. Cancer Res 2004;64:3060-3071.

17. Zhou X, Mok SC, Chen Z, et al. Concurrent analysis of loss of heterozygosity (LOH) and copy number abnormality (CNA) for oral premalignancy progression using the Affymetrix 10K SNP mapping array. Hum Genet 2004;115:327-330. 
18. Beroukhim R, Lin M, Park $Y$, et al. Inferring loss-of-heterozygosity from unpaired tumors using high-density oligonucleotide SNP arrays. PLoS Comput Biol 2006;2:e41. DOl:10.1371/journal.pcbi.0020041.

19. Szanto PA, Luna MA, Tortoledo ME, et al. Histologic grading of adenoid cystic carcinoma of the salivary glands. Cancer 1984;54:1062-1069.

20. He RG, Zhang XS, Zhou XJ. The establishment of cell lines of adenoid cystic carcinoma of human salivary glands (ACC2, ACC 3 ) and a study of morphology. West Chin J Stomatol 1988;6:1-4.

21. Shirasuna K, Watatani K, Furusawa $\mathrm{H}$, et al. Biological characterization of pseudocyst-forming cell lines from human adenoid cystic carcinomas of minor salivary gland origin. Cancer Res 1990;50: 4139-4145.

22. Lin M, Wei LJ, Sellers WR, et al. dChipSNP: significance curve and clustering of SNP-array-based loss-of-heterozygosity data. Bioinformatics 2004;20:1233-1240

23. Li C, Wong WH. Model-based analysis of oligonucleotide arrays: expression index computation and outlier detection. Proc Natl Acad Sci USA 2001;98:31-36.

24. Rumpel CA, Powell SM, Moskaluk CA. Mapping of genetic deletions on the long arm of chromosome 4 in human esophageal adenocarcinomas. Am J Pathol 1999;154:1329-1334.

25. Cerilli LA, Swartzbaugh JR, Saadut R, et al. Analysis of chromosome 9p21 deletion and p16 gene mutation in salivary gland carcinomas. Hum Pathol 1999;30:1242-1246.

26. Uchida K, Oga A, Okafuji M, et al. Molecular cytogenetic analysis of oral squamous cell carcinomas by comparative genomic hybridization, spectral karyotyping, and fluorescence in situ hybridization. Cancer Genet Cytogenet 2006;167:109-116.

27. Zhao $X$, Weir BA, LaFramboise $T$, et al. Homozygous deletions and chromosome amplifications in human lung carcinomas revealed by single nucleotide polymorphism array analysis. Cancer Res 2005;65:5561-5570.

28. Yamamoto $\mathrm{N}$, Mizoe J, Numasawa $\mathrm{H}$, et al. Allelic loss on chromosomes $2 q, 3 p$ and $21 q$ : possibly a poor prognostic factor in oral squamous cell carcinoma. Oral Oncol 2003:39:796-805.

29. Hu N, Flaig MJ, Su H, et al. Comprehensive characterization of annexin alterations in esophageal squamous cell carcinoma. Clin Cancer Res 2004;10:6013-6022.

30. Yang $\mathrm{L}$, Leung $\mathrm{AC}$, Ko JM, et al. Tumor suppressive role of a $2.4 \mathrm{Mb}$ 9q33-q34 critical region and DEC1 in esophageal squamous cell carcinoma. Oncogene 2005;24:697-705.

31. Beder LB, Gunduz M, Ouchida M, et al. Genome-wide analyses on loss of heterozygosity in head and neck squamous cell carcinomas. Lab Invest 2003;83:99-105.

32. Sabbir MG, Roy A, Mandal S, et al. Deletion mapping of chromosome $13 q$ in head and neck squamous cell carcinoma in Indian patients: correlation with prognosis of the tumour. Int J Exp Pathol 2006:87:151-161.

33. Iwaya T, Maesawa C, Kimura T, et al. Infrequent mutation of the human envoplakin gene is closely linked to the tylosis oesophageal cancer locus in sporadic oesophageal squamous cell carcinomas. Oncol Rep 2005:13:703-707.

34. Franzen G, Klausen OG, Grenko RT, et al. Adenoid cystic carcinoma: DNA as a prognostic indicator. Laryngoscope 1991;101:669-673.

35. Franzen $\mathrm{G}$, Nordgard $\mathrm{S}$, Boysen $\mathrm{M}$, et al. DNA content in adenoid cystic carcinomas. Head Neck 1995;17:49-55.
36. Greiner TC, Robinson RA, Maves MD. Adenoid cystic carcinoma. A clinicopathologic study with flow cytometric analysis. Am J Clin Pathol 1989:92:711-720.

37. Maser RS, DePinho RA. Connecting chromosomes, crisis, and cancer. Science 2002;297:565-569.

38. Freier K, Flechtenmacher C, Walch A, et al. Copy number gains on 22 q13 in adenoid cystic carcinoma of the salivary gland revealed by comparative genomic hybridization and tissue microarray analysis. Cancer Genet Cytogenet 2005;159:89-95.

39. Kishi $M$, Nakamura $M$, Nishimine $M$, et al. Loss of heterozygosity on chromosome $6 \mathrm{q}$ correlates with decreased thrombospondin-2 expression in human salivary gland carcinomas. Cancer Sci 2003;94:530-535.

40. Queimado L, Reis A, Fonseca I, et al. A refined localization of two deleted regions in chromosome $6 \mathrm{q}$ associated with salivary gland carcinomas. Oncogene 1998;16:83-88.

41. Yamamoto Y, Virmani AK, Wistuba II, et al. Loss of heterozygosity and microsatellite alterations in p53 and RB genes in adenoid cystic carcinoma of the salivary glands. Hum Pathol 1996;27: 1204-1210.

42. Chan K-L, Lee J, Guan X-Y, et al. High-density allelotyping of chromosome $8 p$ in hepatocellular carcinoma and clinicopathologic correlation. Cancer 2002;94:3179-3185.

43. lacobuzio-Donahue CA, van der Heijden MS, Baumgartner MR, et al. Large-scale allelotype of pancreaticobiliary carcinoma provides quantitative estimates of genome-wide allelic loss. Cancer Res 2004;64:871-875.

44. Fourouclas N, Campbell PJ, Bench AJ, et al. Size matters: the prognostic implications of large and small deletions of the derivative 9 chromosome in chronic myeloid leukemia. Haematologica 2006;91:952-955.

45. Lopez-Gines C, Cerda-Nicolas M, Llombart-Bosch A. Cytogenetic findings in a new case of adenoid cystic carcinoma arising in sphenoidal sinus. Cancer Genet Cytogenet 1994;75:150-152.

46. Higashi $K$, Jin $Y$, Johansson $M$, et al. Rearrangement of $9 p 13$ as the primary chromosomal aberration in adenoid cystic carcinoma of the respiratory tract. Genes Chromosomes Cancer 1991;3:21-23.

47. Maruya $\mathrm{S}$, Kurotaki $\mathrm{H}$, Shimoyama $\mathrm{N}$, et al. Expression of p16 protein and hypermethylation status of its promoter gene in adenoid cystic carcinoma of the head and neck. ORL J Otorhinolaryngol Relat Spec 2003;65:26-32.

48. Nishimine $M$, Nakamura $M$, Kishi $M$, et al. Alterations of p14ARF and p16INK4a genes in salivary gland carcinomas. Oncol Rep 2003;10:555-560.

49. Yamamoto $Y$, Saka T, Makimoto $K$, et al. Histological changes during progression of adenoid cystic carcinoma. J Laryngol Otol 1992;106:1016-1020.

50. Nagao T, Gaffey TA, Serizawa $H$, et al. Dedifferentiated adenoid cystic carcinoma: a clinicopathologic study of 6 cases. Mod Pathol 2003;16:1265-1272.

51. Papadaki H, Finkelstein SD, Kounelis $\mathrm{S}$, et al. The role of p53 mutation and protein expression in primary and recurrent adenoid cystic carcinoma. Hum Pathol 1996;27:567-572.

52. Yamamoto $Y$, Wistuba II, Kishimoto $Y$, et al. DNA analysis at p53 locus in adenoid cystic carcinoma: comparison of molecular study and p53 immunostaining. Pathol Int 1998;48:273-280. 